Published in final edited form as:

Surg Oncol Clin N Am. 2019 July ; 28(3): 447-464. doi:10.1016/j.soc.2019.02.002.

\title{
Immunotherapy in ovarian cancer
}

Weimin Wang, PhD [Research lab specialist], Department of Surgery, University of Michigan School of Medicine, Ann Arbor, Michigan, USA

\author{
J. Rebecca Liu, MD [Associate Professor], \\ Department of Obstetrics and Gynecology, University of Michigan School of Medicine, Ann Arbor, \\ Michigan, USA
}

Weiping Zou, MD, PhD [Charles B. de Nancrede Professor of Surgery, Immunology and Biology, Co-Director]

Tumor Immunology and Transplantation Biology Program, University of Michigan School of Medicine, Ann Arbor, Michigan, USA

\section{Keywords}

Immunotherapy; ovarian cancer; checkpoint blockade; adoptive T-cell transfer; cancer vaccine

\section{Introduction}

\begin{abstract}
Epithelial ovarian cancer (EOC) is the most common cause of gynecological cancerassociated death in woman ${ }^{1}$. To date, there are no effective means of screening or early detection for this disease, and consequently, the majority of patients have advanced stage disease involving metastasis at the time of initial diagnosis ${ }^{2}$. The first-line therapy of EOC includes surgical debulking and platinum-based chemotherapy ${ }^{3}$. Although initial response is often excellent, patients oftentimes develop resistance to chemotherapy and succumb to their disease. Therefore, it is critical to develop novel treatment strategies to improve clinical outcomes.

Current immunotherapy, including checkpoint blockade and adoptive T-cell transfer (ACT), has become a clinically effective treatment modality for a wide variety of cancer types. However, the application of immunotherapy in EOC is still being tested in clinical trials. Tumor-infiltrating lymphocytes (TILs), positively correlating with patient survival, have now been recognized as a predictive biomarker for immunotherapy and chemotherapy responses ${ }^{3,4}$. In ovarian cancer, the presence of $\mathrm{CD}^{+} \mathrm{T}$-cell and its correlation with improved clinical outcome had been observed in an early study, which establishes the validity of utilizing immunotherapy for EOC management ${ }^{5}$. Later studies demonstrated the
\end{abstract}

CORRESPONDING AUTHOR Weiping Zou, BSRB 5478, 109 Zina Pitcher Place, Ann Arbor MI 48109-0669, wzou@med.umich.edu.

Publisher's Disclaimer: This is a PDF file of an unedited manuscript that has been accepted for publication. As a service to our customers we are providing this early version of the manuscript. The manuscript will undergo copyediting, typesetting, and review of the resulting proof before it is published in its final citable form. Please note that during the production process errors may be discovered which could affect the content, and all legal disclaimers that apply to the journal pertain. 
prognostic significance of $\mathrm{FOXP} 3^{+} \mathrm{T}_{\text {reg }}$ cells ${ }^{6}$ and/or the ratio of $\mathrm{CD} 8^{+} \mathrm{T}$ cells versus $\mathrm{FOXP}^{+} \mathrm{T}_{\text {reg }}$ cells in ovarian cancer, and confirmed CD $8^{+} \mathrm{T}$ cells as important antitumor effectors ${ }^{6,7}$. CD8 ${ }^{+}$effector $\mathrm{T}$ cells, are poised to eliminate tumor cells after recognizing tumor associated antigens and particularly the neoantigens presented on the surface of tumor cells ${ }^{8}$. However, their activation and effector functions are often held in check and attenuated by inhibitory signals in the tumor microenvironment, which include inhibitory cytokines, immunosuppressive metabolites, and immunoinhibitor B7 family membersexpressed on the antigen presenting cells (APCs) and tumor cells ${ }^{9,10}$. Checkpoint blockade can restore the antitumor activity of effector $\mathrm{T}$ cells and mediate tumor regression ${ }^{11}$. Adoptive transfer of ex vivo-expanded tumor-specific $\mathrm{T}$ cells can directly increase the number of $\mathrm{CD} 8^{+}$effector $\mathrm{T}$ cells in tumor ${ }^{12}$. Cancer vaccine may activate TAA-specific $\mathrm{CD} 8^{+} \mathrm{T}$ cell response through $\mathrm{APCs}^{13}$. These immunotherapeutic strategies have been tested or are under development in ovarian cancer. The progress of immunotherapy in EOC will be discussed in this review.

\section{Checkpoint blockade}

Several checkpoint molecules have been identified, including cytotoxic T-lymphocyteassociated antigen 4 (CTLA-4), PD-1, and PD ligand 1 (PD-L1, B7-H1). Antibodies targeting these checkpoints can block their inhibitory function, thus unleashing the antitumor activity of effector T cells and mediating tumor regression ${ }^{14}$. There are currently 6 immune checkpoint inhibitors approved by the Food and Drug Administration (FDA): one CTLA-4 antibody (Ipilimumab), two PD-1 antibodies (Pembrolizumab and Nivolumab) and three PD-L1 antibodies (Avelumab, Atezolizumab and Durvalumab). These antibodies have demonstrated remarkable clinical benefits in a wide range of tumor types, such as melanoma, NSCLC, renal cell and urothelial cancer ${ }^{11}$.

Although anti-CTLA-4 and anti-PD-1/PD-L1 both enhance anti-tumor immune response through increasing the number of tumor-infiltrating $\mathrm{T}$ cells and recovering the effector function of cytotoxic $\mathrm{T}$ cells, their underlining cellular and molecular mechanisms are distinct. CTLA-4 is a receptor on T cells and shares the same set of ligands with costimulatory receptor $\mathrm{CD} 28$ but with a much higher affinity, therefore providing a competitive inhibition ${ }^{15}$. Antibody targeting CTLA-4 blocks the ligation of CTLA-4 with its ligands, which prevents inhibitory signal transduction and results in increased CD28mediated co-stimulation. CTLA-4 blockade has been shown to directly enhance the proliferation and activation of tumor-specific $\mathrm{CD} 8^{+} \mathrm{T}$ cells ${ }^{16}$.

PD-1 is another inhibitory receptor expressed on T cells. Unlike CTLA-4, which regulates Tcell activation at the priming phase, PD-1 inhibits effector T-cell activity mainly in the effector phase in peripheral tissue and tumor microenvironment ${ }^{17}$. Upon activation by its ligands PD-L1 or PD-L2, PD-1 becomes phosphorylated and recruits the inhibitory phosphatases SHP-2, which rapidly dephosphorylates CD28 and inactivates the costimulatory signaling ${ }^{18}$. Of the two ligands, PD-L1 is considered to be more relevant in the tumor microenvironment because of its expression on tumor, stromal and immune cells ${ }^{19}$. Antibodies targeting either PD-1 or PD-L1 result in abrogation of the negative signal, thus restoring the function of tumor-infiltrating $\mathrm{T}$ cells and mediating tumor regression ${ }^{20}$. 


\section{Single-agent checkpoint inhibitor in ovarian cancer}

Several antibodies targeting PD-1, PD-L1 and CTLA-4 have been tested in patients with recurrent ovarian cancer. Overall, objective response (OR) (complete response (CR) + partial response (PR)) rates range from $5.9 \%$ to $15 \%$ (Table 1 ).

In an early phase I trial, 207 patients with selected advanced cancers, including 17 with EOC, received anti-PD-L1 antibody (BMS-936559). Among the 17 women, 1 (5.9\%) had a PR and a total of 4 women $(23 \%)$ had disease control. This is the first data suggesting immune checkpoint blockade as a potentially valuable therapeutic approach in ovarian cancer $^{21}$. Later, a phase II trial of an anti-PD1 antibody (nivolumab) was conducted in 20 patients with platinum-resistant EOC. 2 patients experienced a CR, 1 had a PR and 6 had a stable disease (SD), leading to a disease control rate of $45 \%$. Pembrolizumab, another antiPD1 antibody, was shown to have an OR rate of $11.5 \%$ and a disease control rate of $34.6 \%$ in 26 patients with advanced EOC. Patients were selected with a PD-L1 expression level of $\geq 1 \%$ of tumor cells for trial entry, and $85 \%$ of patients were heavily pre-treated ${ }^{23}$.

Atezolizumab is a fully humanized monoclonal antibody against PD-L1 and has been approved for metastatic non-small cell lung cancer (NSCLC) treatment. In a phase I study (), 12 patients with recurrent ovarian cancer were treated with atezolizumab until loss of clinical benefit. Of 9 response-evaluable patients, 2 patients (22\%) had a PR and 5 patients had PD. PD-L1 status on immune cells (IC) was found to correlate with response ${ }^{24}$. More recently, a study assessed the safety and activity of another anti-PD-L1 antibody (avelumab) in 124 women with recurrent or refractory EOC (). This is the largest study of anti-PD(L)1 agents in patients with EOC to date. Avelumab achieved an OR of $9.7 \%$ and a disease control rate of $54.0 \%$. Again, in this cohort more than two-thirds of the patients had received previous lines of therapy. This study showed that single-agent avelumab was clinically active in heavily pretreated patients with $\mathrm{EOC}^{25}$.

In addition to $\mathrm{PD}(\mathrm{L}) 1$ blockade, the activity and safety of anti-CTLA- 4 antibody in EOC was studied. In a phase II trial, 40 patients with recurrent platinum-sensitive EOC received an anti-CTLA-4 antibody ipilimumab (). The OR rate was $10.3 \%$ and adverse events (AEs) of grade $>3$ occurred in $50 \%$ of the patients ${ }^{26}$.

In summary, these studies suggest that single-agent checkpoint inhibitor, in particular $\mathrm{PD}(\mathrm{L})-1$ blockade, is promising in epithelial ovarian cancer, although the OR rate is much lower compared with the response rates achieved in melanoma, NSCLC, renal cell and urothelial cancer. Although potential mechanisms of resistance toward immune checkpoint blockade are under investigation, combining checkpoint inhibitors with other biological agents to enhance the anti-tumor immune response has been rationally illustrated and numerous combinatorial trials have entered the clinic. The combination of two checkpoint blockades (nivolumab + ipilimumab) was firstly tested in melanoma ${ }^{27}$. In EOC, the same combination () and another combination of durvalumab (anti-PD-L1) and tremelimumab (anti-CTLA-4) () are both being investigated. 


\section{Combination with PARP inhibitor}

Tumor mutational burden (TMB) is a recently suggested biomarker of response to $\mathrm{PD}(\mathrm{L})-1$ blockade therapy ${ }^{28}$. Tumor-specific mutations can result in immunogenic neoantigens, therefore high TMB correlates with high neoantigen load. This higher neoantigen load correlated with an increased number of tumor-infiltrating lymphocytes (TILs), which is counterbalanced by PD-1/PD-L1 signaling ${ }^{29}$. Melanoma, lung cancer and a subtype of colorectal cancer with mismatch repair deficiency have a significantly higher TMB, and they all have a remarkable response to PD- 1 inhibition ${ }^{30}$. In ovarian cancer roughly the $25-30 \%$ of the high grade serous (HGS) harbor a mutation in the BRCA1 or BRCA2 genes, which have integral functions in DNA homologous recombination (HR) repair. The presence of a HR-deficiency is associated to a higher mutation burden and increased TILs compared to the BRCA-wild type tumors ${ }^{31}$. Thus, BRCA-mutated HGS ovarian cancer could represent a subtype with potential increased response to immune checkpoint blockade alone or in combination with poly (ATP-ribose) polymerases (PARP) inhibitors. Several clinical trials evaluating the combination of Olaparib (PARP inhibitor) and checkpoint blockade in patients with BRCA-mutated ovarian cancer are currently ongoing (Table 2). An ongoing phase II trial (), the combination of olaparib and durvalumab (anti-PD-L1) was found to induce objective responses in over $70 \%$ of patients with relapsed, platinum-sensitive, $B R C A$-mutated ovarian cancer $^{32}$. Another phase I/II trial evaluating the combination Olaparib with anti-CTLA-4 and anti-PD-L1 in patients with BRCA1/BRAC2-mutated EOC () is also ongoing.

\section{Combination with VEGF inhibitor}

Another interesting novel approach is to combine vascular endothelial growth factor (VEGF) inhibitor and checkpoint blockade. VEGF is primarily known as a mediator of angiogenesis; however, emerging studies have also recognized VEGF as a critical mediator of immune suppression in the tumor microenvironment ${ }^{33}$. Inhibitors targeting VEGF or VEGF receptors (VEGFR) have been shown to decrease the numbers of regulatory $\mathrm{T}$ cells and myeloidderived suppressor cells (MDSC) and enhance the infiltration of effector T cells ${ }^{33,34}$. Several clinical trials are currently ongoing to evaluate the safety and efficacy of the combination of bevacizumab (anti-VEGF) and checkpoint blockade in melanoma, NSCLC and renal cell cancer. For EOC, a phase II trial focused on platinum-resistant disease, in which atezolizumab (anti-PD-L1) is given together with bevacizumab and acetylsalicylic acid (). In another study, durvalumab combined with olaparib or the VEGFR inhibitor cediranib in EOC is being tested ().

\section{Combination with chemotherapy}

Chemotherapy can be used in combination with checkpoint blockade to amplify the antitumor immune response. In addition to direct cytotoxic effects on tumor cells, a proportion of chemotherapy agents may actually induce immunogenic cell death, expand neoantigen repertoire, increase antigen presentation, change the inflammatory milieu of tumor microenvironment and/or decrease the numbers of immunosuppressive cells, thereby inducing or enhancing the anti-tumoral immune response ${ }^{35,36}$. There are now more than 200

Surg Oncol Clin N Am. Author manuscript; available in PMC 2020 July 01. 
clinical trials testing immune checkpoint inhibitors in combination with various chemotherapeutic agents across different cancer types.

In ovarian cancer, there are currently 5 randomized phase III trials adding PD-L1 blockade to chemotherapy: 2 are with avelumab and 3 are with atezolizumab. JAVELIN Ovarian 200 () is the first one to compare avelumab alone or in combination with pegylated liposomal doxorubicin in patients with platinum-resistant/refractory recurrent ovarian, fallopian tube or peritoneal cancer. The second trial is JAVELIN Ovarian 100 (), which will access the efficacy of avelumab in combination with conventional chemotherapy carboplatin and paclitaxel in patients with previously untreated ovarian cancer. The other 3 trials with atezolizumab involve adding both chemotherapy and bevacizumab (anti-VEGF) to atezolizumab. The trial will assess the efficacy of atezolizumab in combination with bevacizumab plus platinum-based chemotherapy in patients with ovarian cancer who have platinum-sensitive relapse (platinum-free interval > 6 months). The trial will test the activity of atezolizumab in combination with bevacizumab paclitaxel and carboplatin in participants with newly diagnosed ovarian cancer. The trial combines atezolizumab with bevacizumab and pegylated liposomal doxorubicin in platinum resistant ovarian cancer.

Similarly, another PD-L1 inhibitor Durvalumab and a PD-1 inhibitor in combination with chemotherapy are also being tested in several phase I/II clinical trials in ovarian cancer. These include Durvalumab combined with PLD (), Pembrolizumab combined with paclitaxel and carboplatin () or with dose dense paclitaxel ().

Based on the published results and preliminary data, combinational therapy including PD-1/PD-L1 inhibitors and chemotherapy certainly has the potential to be superior to chemotherapy alone ${ }^{37,38}$. However, there are some ongoing challenges. Cytotoxic chemotherapy has historically been considered immunosuppressive because most chemotherapeutic agents indiscriminately impair cellular division and thus impact not only tumor cells, but also effector lymphocytes and innate leukocytes ${ }^{39}$. Moreover, recent studies demonstrated that certain chemotherapy agents might attenuate the anti-tumor immune response $\mathrm{e}^{40,41}$. Therefore, to thoroughly assess the combination with chemotherapy, selection of chemotherapy agents, optimization of the sequencing, timing, dose, and management of concurrent toxicities will be required.

\section{Adoptive T-cell therapy (ACT)}

Adoptive T cell therapy consists of the infusion of ex vivo-expanded tumor-specific T cells that can recognize and attack tumor cells resulting in tumor regression ${ }^{12}$. There are currently two major forms of ACT being used for cancer treatment: isolating and expanding tumorinfiltrating lymphocytes (TIL) or using genetically modified $\mathrm{T}$ cells that express a specific TCR or a chimeric antigen receptor $(\mathrm{CAR})^{42}$. Both strategies have been tested in preclinical ovarian cancer models, and currently being investigated in early phase trials.

\section{TIL therapy}

TIL therapy has been shown to induce long-lasting and complete regression of metastatic tumor in patients with melanoma ${ }^{43}$. Generally, ACT of TIL consists of several steps: 
1. isolation of TILs from fresh tumor biopsy;

2. selection and expansion of tumor-specific T-cell population ex vivo;

3. lymphodepletion of immunosuppressive components by chemotherapy or radiation; and

4. infusion of expanded T cells to patients with IL-2 administration.

During this long-term procedure, both $\mathrm{CD}^{+}$and $\mathrm{CD} 8^{+} \mathrm{T}$ cells are expanded ex-vivo and are subsequently infused back into the patient ${ }^{44}$.

TIL therapy in melanoma response rates can be from $47 \%$ to $72 \% 45$. The efficacy of TIL therapy in EOC has also been investigated in several early clinical studies (Table 3). The first trial of TIL therapy in EOC enrolled 17 patients with advanced or recurrent platinumresistant disease. Among 7 patients received TIL infusion them, $1 \mathrm{CR}$ and 4 PR were reported and tumor regression lasted for 3-5 months. Another group of 10 patients received TIL infusion combined with cisplatin-based chemotherapy. 7 had a CR, 2 had a PR, and 1 had a SD. The duration of response ranged from 13 to more than 26 months $^{46}$. Later, a pilot study was conducted to determine the feasibility and clinical effects of TIL infusion plus low-dose IL-2 in patients with platinum-resistant ovarian cancer. Among 8 patients received the treatment: 2 had reduction in ascites, 1 had reduction in tumor burden and CA-125 and 1 had surgically confirmed stable disease ${ }^{47}$. In 1995, Fujita et. al tested the efficacy of maintenance therapy with TIL in ovarian cancer patients with no evidence of disease after debulking surgery and adjuvant platinum-based chemotherapy. 13 patients received TIL and 11 additional patients (control group) received standard treatment. The TIL group had significant improved 3-year OS $(100 \%$ vs $65 \%, \mathrm{p}<0.01)$ and 3-year disease free survival (DFS) $(82.1 \%$ vs $54.5 \%, \mathrm{p}<0.05)$ compared to the control group ${ }^{48}$. In summary, these early trials showed limited efficacy of TIL therapy in EOC. However, it is notable that none of these trials used current chemotherapy regimens for lymphodepletion before TIL infusion, and the methods for ex-vivo TIL expansion have changed considerably. Therefore there is still significant biological and clinical rationale to test TIL therapy in ovarian cancer (Table 4). An ongoing phase II trial is assessing the anti-tumor efficacy of TIL in metastatic digestive tract, urothelial, breast, or ovarian/endometrial cancer (). In this study, short-term cultured autologous TIL will be used after a lymphocyte depleting regimen, and checkpoint blockade pembrolizumab will be administrated at time of progression. More recently, a phase I study focused upon patients with platinum resistant high-grade serous ovarian, fallopian tube, or primary peritoneal cancer () was opened. Patients will receive an infusion of autologous TIL which will be stimulated ex-vivo with autologous dendritic cells (DCs) and OKT3 (anti-CD3 antibody). This "re-stimulation" is believed to make autologous TIL work better.

\section{TCR-engineered T cells}

Autologous T cells can be genetically modified to express a cloned TCR directed towards a specific antigen. $T$ cells isolated from patient peripheral blood are used for transduction with a viral vector encoding TCR, and then can rapidly be expanded to a great number for infusion within a short time. Similarly to the endogenous TCR-expressed T cells, TCR-

Surg Oncol Clin N Am. Author manuscript; available in PMC 2020 July 01. 
engineered T cells recognize tumor-specific antigen in a MHC-dependent manner and its function can be negatively regulated by the immunosuppressive signals in the tumor microenvironment ${ }^{49}$.

The choice of antigen to be targeted determines the specificity of TCR-engineered T cells. To date, most of the studies have engineered TCRs to target tumor-associated antigens, such as Melan-A/MART-1 and gp100, or cancer germline antigens such as NY-ESO-1 and MAGE $^{50,51}$. Although these TCR-engineered T cells have resulted in objective responses in the majority of treated patients, especially in melanoma patients, they also caused "offtumoron-target" toxicity by reacting to healthy organs that expressed the targeted antigen ${ }^{52}$. In EOC, so far, there are no completed clinical trials using TCR-engineered T cells and several phase I studies are on-going (Table 4). The study evaluates the safety and effect of MAGE-A4-specific TCR gene transduced T cells in HLA-A*24:02 positive patients with refractory ovarian cancer that express the antigen MAGE-A4. Another 3 trials (, , ) all use T cells engineered with NY-ESO-1-specific TCR to treat ovarian cancer patients with the HLA-A*02:01 or HLA-A*02:06 allele and whose tumor expresses the NY-ESO-1 antigen. NY-ESO-1 is highly expressed in various types of cancer, including EOC. While in normal somatic tissues its expression is restricted to the germline cells, which lack HLA molecules and cannot be recognized by T cells. Therefore, NY-ESO-1 has been selected as an attractive target for ACT in clinical trials. Most recently, efforts have been focused on the development of T cells engineered with TCRs specific to particular tumor neoantigens. A new pilot study has demonstrated the feasibility of the process from identification of neoantigen to production of the neoantigen-specific cytotoxic TCR-engineered T cells for ovarian cancer $^{53}$.

\section{CAR T cells}

CAR is another mean for providing recognition specificity to engineered T cells. CAR molecule combines the antigen-binding domain of a single-chain variable fragment ( $\mathrm{scFv}$ ) derived from an antibody with cytoplasmic signaling motifs. The $\mathrm{scFv}$ fragment recognizes antigens that present on tumor cell surface. The cytoplasmic signaling motifs mediate T-cell activation, consisting of a CD3-zeta activation domain and two co-stimulatory domains, CD28 and CD137/4-1BB. Upon antigen encounter, intracellular domains of the CAR transduce the activation signals, leading to T-cell activation with cytotoxic function ${ }^{54,55}$. Recently, the first two CAR T-cell therapies (axicabtagene ciloleucel and tisagenlecleucel) have been approved for the treatment of refractory diffuse large B-cell lymphoma (DLBCL) and refractory B-cell precursor acute lymphoblastic leukemia (ALL). They both use antiCD19-redirected CAR T cells and have achieved impressive response rates. Thus, the field of CAR T-cell therapy is now booming and over 200 CAR-T clinical trials are running.

In EOC, the first study of adoptive transfer of folate receptor-a (FRa)-specific CAR T cells in ovarian cancer patients showed no clinical benefit probably because of low expression of the CAR and poor persistence of the transferred T cells ${ }^{56}$. Another phase I trial () tested the mesothelin-specific CAR T cells in six recurrent serous ovarian cancer patients. The treatment was feasible and safe and trafficking of CAR T cells to the tumor site was observed. All six patients showed stable disease after one month treatment ${ }^{57}$. The effect of 
mesothelin-specific CAR T cells is promising and being tested in two more phase I/II clinical trials (, ) in mesothelin expressing cancers, including ovarian cancer. In addition to FRa and mesothelin, some other CAR T cells targeting tumor-associated antigens are under investigation in clinical trials, including Mucin-16 (), Epidermal growth factor receptor (EGFR) (), Human epidermal growth factor receptor-2 (HER-2) (), CD133 () and Natural killer group 2D (NKG2D) (). Identifying the tumor-specific surface antigens is the priority to manufacture effective CAR T cells against solid tumors. Moreover, the immunosuppressive environment within a solid tumor also inhibits the trafficking and effector function of CAR T cells. Therefore, various combinations of CAR T cells with other therapies would be expected to achieve greater persistence and response.

\section{Cancer vaccine}

The biological principle of cancer vaccines is to provoke a tumor-specific immune response that involves both innate and adaptive immunity. Therapeutic cancer vaccine theoretically could increase TAA presentation by APCs and then generate TAA-specific CD8+ T cells to eradicate tumor cells. Moreover, vaccine-induced immune response has the ability of immunological memory that can persist for long periods of time ${ }^{58}$. In EOC, many clinical studies have revealed induced antigen-specific $\mathrm{T}$ cell response and improved survival in different degrees by using different vaccines, including recombinant protein or peptide vaccines and whole tumor lysate or dendritic cell (DC)-based autologous vaccines.

\section{Protein/peptide-based vaccine}

Protein/peptide-based vaccines are usually based on the well-defined TAAs and administered together with an adjuvant to improve their presentation by endogenous DCs. The ideal TAA should be selective expression by tumor cells relative to normal tissue and high immunogenicity. Among antigens targeted for vaccination, NY-ESO-1 is one of the most spontaneously immunogenic tumor antigens that have been tested in clinical trials in various solid tumors, including ovarian cancer ${ }^{59}$. Vaccination of HLA-A*0201-positive EOC patients with NY-ESO-1 peptide (NY-ESO-1 157-165) and Montanide, an incomplete Freund's adjuvant, induced specific T-cell immune response in patients with both NYESO-1-positive and NY-ESO-1-negative tumors ${ }^{60}$. Another study used the peptide NYESO-1 157-170 that contains both CD4+ and CD8+ T cell epitopes for vaccination in advanced EOC patients. Long-lived and functional vaccine-elicited CD4+ and CD8+ T cells were detected in patients with no evidence of disease (NED) for $>6$ months $^{61}$. Overlapping long peptides (OLP) derived from NY-ESO-1 was also tested in various adjuvant combinations in patients with EOC. NY-ESO-1-specific antibody and CD8+ T cell responses were detected in 6 of $13(46 \%)$ and 8 of $13(62 \%)$ of patients after vaccination with OLP+ Montanide, and both were detected in 10 of 11 (91\%) of patients receiving OLP + Montanide+ poly-ICLC ${ }^{62}$. Recombinant poxviruses (vaccinia and fowlpox) expressing the whole protein of NY-ESO-1 was evaluated in a phase II clinical trials in 22 ovarian cancer patients. Integrated NY-ESO-1-specific antibody and CD4+ and CD8+ T cells were induced in a high proportion of EOC patients ${ }^{63}$. The expression of NY-ESO-1 is suppressed by DNA methylation and can be enhanced by the DNA methyltransferase (DNMT) inhibitor decitabine. A phase I trial combined the NY-ESO-1 peptide vaccine with escalating doses of 
decitabine and doxorubicin liposome chemotherapy in 12 patients with relapsed EOC.

Increased NY-ESO-1-specific antibodies and $\mathrm{CD} 8^{+} \mathrm{T}$ cells were detected in the majority of patients. Among 10 evaluable patients, 5 patients had SD and 1 had a $\mathrm{PR}^{64}$. Other antigens such as p53, Her2 and FRa have also been tested for vaccination in clinical studies of EOC. A p53 synthetic long peptide (p53-SLP) vaccine combined with low-dose cyclophosphamide was tested in a phase 2 single-arm trial in EOC. p53-specific effector T cells were observed in $90 \%$ of patients and stable disease was observed in $20 \%$ of patients ${ }^{65}$. Many different peptides derived from Her2 have also been tested in ovarian cancer. However, no immunogenicity was observed in most studies using single or mixed Her2 peptides and no clinical data was obtained ${ }^{66}$. FRa peptide vaccine was tested in a recent phase I clinical trial enrolling patients with EOC who showed no evidence of disease after conventional therapy. Vaccination was well tolerated in all patients and induced immune response in more than $90 \%$ of patients ${ }^{67}$.

\section{DC-based vaccine}

DC is the most potent APC population for activating anti-tumor T-cell response. Also because it rarely presents immune-related toxicities, DC has become the most frequently used cellular therapeutic in clinical trials. DC can be given alone after cytotoxic chemo- or radiotherapy or loaded ex vivo with various antigens, such as proteins, peptides or the whole tumor lysate, before reinfusion into patients ${ }^{68}$.

\section{Protein- or peptide-loaded DC}

Similar with recombinant protein/peptide vaccine, this strategy is also based on well-defined TAAs. Autologous DCs mare pulsed with recombinant protein or peptides before reinfusion. An early phase I study evaluated immunization with autologous DC pulsed with mannanMUC1 fusion protein to treat patients with advanced cancer, including 2 EOC patients. T cell response was observed in all patients and 1 of 2 EOC patients in progression at entry was stable after therapy for at least 3 years ${ }^{69}$. Later, Lapuleucel-T, the Her-2-GM-CSF fusion protein loaded DC vaccine was tested in 4 patients with Her2-postive ovarian cancer. Her-2-specific T cell response was detected after vaccination and 2 patients had $\mathrm{SD}^{70}$. Peptide-sloaded DC vaccines were also tested in ovarian cancer. A phase I trial enrolled 10 patients including 3 EOC patients to receive immunization with DC pulsed with Her-2- or MUC-1-derived peptides. Antigen-specific T cell responses were detected in 5 of 10 patients ${ }^{71}$. In another phase I trial, 11 EOC patients were immunized with DC loaded with Her2, hTERT and PADRE peptides with low-dose cyclophosphamide. Her2- and hTERTspecific T-cell responses were observed and the 3 -year overall survival was $90 \% 72$.

\section{Whole tumor lysate-loaded DC}

The advantage of whole tumor cells as a source of antigens for DC based vaccine is that the entire repertoire of antigens associated with a particular tumor, including the specific neoantigens, may be processed and presented to the immune system. Therefore, this strategy is predicted to generate a better immune response and prevent tumor immune escape through antigen-loss variants or mutations. An earlier phase I study used DC loaded with crude 
whole tumor lysate to treat 8 patients, including 6 with recurrent EOC. 3 patients showed stable disease lasting 25 to 45 weeks and tumor antigen specific immunity was detected in 2 of 6 evaluable patients ${ }^{73}$. Later, oxidized whole tumor lysate with enhanced immunogenicity were used to load DC. A recent pilot trial tested the autologous oxidized lysate-loaded autologous DC in recurrent ovarian cancer patients. DC vaccine was administered alone (cohort $1, \mathrm{n}=5$ ), in combination with bevacizumab (cohort $2, \mathrm{n}=10$ ), or bevacizumab plus low-dose cyclophosphamide (cohort 3, $\mathrm{n}=10$ ). 3 patients had SD in cohort 1; 1 patient had PR and 4 patients had SD in cohort 2; and in cohort 3,1 patient had PR and 6 patients had $\mathrm{SD}$. Vaccination induced $\mathrm{T}$ cell responses to tumor antigen, which were associated with significantly prolonged survival ${ }^{74}$.

The FANG vaccine is another whole tumor cell-based vaccine. Autologous tumor cells are eletroporated with FANG vector, a plasmid expressing GM-CSF and a bifunctional shRNA against furin ${ }^{75}$. This vaccine has been tested in a Phase I study recruiting 27 patients with advanced cancer, including 5 ovarian cancers ${ }^{75}$. Currently, this vaccine is being tested in a phase II study in patients with high-risk stage III/IV ovarian cancer ().

\section{Conclusion and future directions}

\section{Checkpoint blockade combination therapy}

To date, checkpoint blockade is the most promising and studied immunotherapy in ovarian cancer. The combinations of PD(L)- 1 antibodies with PARP inhibitors or conventional chemotherapy have achieved good response in clinical trials and demonstrate excellent clinical potential. Tumor cells create an immunosuppressive microenvironment through multiple mechanisms ${ }^{10,76,77}$, therefore combinatorial therapeutic strategies will be required to unleash the maximal antitumor immune response. Epigenetic modulators, including the inhibitors targeting HDAC, enhancer of zeste homolog 2 (EZH2), DNA methyltransferase 1 (DNMT1) and histone demethylase LSD1 are recently found to have the ability to regulate the anti-tumor immune response ${ }^{78-80}$. It has been showed that inhibitors of EZH2 and DNMT1 could induce chemokine expression in tumor cells, increase CD8+ T cells tumor infiltration and augment antitumor efficacy of PD-L1 blockade in an EOC preclinical model $^{78}$. Two ongoing clinical trials in ovarian cancer are testing the combinations of PD-1 blockade pembrolizumab with epigenetic modulators: one is a DNMT1 inhibitor guadecitabine () and another is a DNA methyltransferase inhibitor azacitidine (). Targeting tumor metabolism represents another promising strategy to combine with PD blockade. Growing evidence has shown that the tumor microenvironment supports metabolic reprogramming that dampens $\mathrm{T}$ cell function. Indoleamine 2,3-dioxygenase (IDO)-mediated tryptophan catabolism has been recognized as a mechanism of immunosuppression in many types of cancer, including EOC. It has been well established that IDO inhibition could enhance antitumor immune response ${ }^{81}$. A current clinical trial involving EOC patients is assessing the combination between IDO inhibitor epacadostat and PD-L1 blockade nivolumab.

In addition to the PD-L1/PD-1 axis, other immunosuppressive molecules, such as T cell immunoglobulin mucin 3 (Tim-3), lymphocyte activation gene 3 protein (LAG3) and T cell immunoglobulin and ITIM domain (TIGIT), are all potential targets for developing 
immunotherapy ${ }^{82}$. The combinations of PD blockade with LAG3 blockade are under testing in clinical trials ().

\section{Neoantigen vaccine}

Another promising approach is the development of EOC neoantigen vaccine. Cancer neoantigens are a class of HLA-bound peptides that are generated by tumor missense mutations. They are exclusively tumor-specific and highly immunogenic, thus can be recognized by host immune system ${ }^{83}$. Neoantigens-derived vaccine has been recently tested in several clinical studies in patients with melanoma. The vaccine consisting of synthesized long peptides targeting up to 20 neoantigens per patient plus poly-ICLC (Hiltonol) was evaluated in a phase I study in patients with advanced cutaneous melanoma ${ }^{84}$. Vaccineinduced both $\mathrm{CD}^{+}$and $\mathrm{CD}^{+} \mathrm{T}$ cells were detected against unique neoantigens. In 6 immunized patients, 4 had no recurrence at 25 months after vaccination. 2 recurrent patients were further treated with PD1 blockade and experienced complete tumor regression. In another study, a RNA-based poly-neo-epitope vaccine was tested in patients with advanced cutaneous melanoma ${ }^{85}$. Similarly, both $\mathrm{CD}^{+}$and $\mathrm{CD} 8^{+} \mathrm{T}$-cell responses were detected against $66 \%$ and $25 \%$ of the candidate neoepitopes, respectively. These studies indicate that neoantigen vaccine can induce expansion of the repertoire of neoantigen-specific $\mathrm{T}$ cells and mediate tumor regression, which represents a novel strategy of personalized immunotherapy for patients with cancer, including EOC.

In summary, the tumor microenvironment is the main battleground between tumor cells and the host immune system. Major immunosuppressive networks have been identified in the human epithelial ovarian cancer microenvironment. Current checkpoint therapy targets the cancer immunosuppressive mechanisms and generates clinical therapeutic efficacy in a fraction of patients. However, it seems that EOC patients are relatively insensitive to the single checkpoint therapy. Nonetheless, tumor associated effector $\mathrm{T}$ cells are positively associated with ovarian cancer patient survival and response to chemotherapy ${ }^{3}$. As effector $\mathrm{T}$ cell tumor trafficking is controlled by the cancer epigenetic pathway and $\mathrm{T}$ cell effector function may be additionally affected by metabolic pathway, the combination therapy by targeting the immunosuppressive networks (e.g. PD-L1/PD-1 pathway) and qualitatively supporting specific $\mathrm{T}$ cells may be a potential successful immunotherapy approach for ovarian cancer patients.

\section{Acknowledgments}

\section{DISCLOSURE STATEMENT}

This work was supported in part by research grants from the NIH/NCI R01 grants (W. Z) (CA217648, CA123088, CA099985, CA193136 and CA152470) and the NIH through the University of Michigan Roger Cancer Center Support Grant (CA46592).

\section{References}

1. Jayson GC, Kohn EC, Kitchener HC, Ledermann JA. Ovarian cancer. Lancet. 1011 2014;384(9951):1376-1388. [PubMed: 24767708]

2. Ledermann JA, Raja FA, Fotopoulou C, et al. Newly diagnosed and relapsed epithelial ovarian carcinoma: ESMO Clinical Practice Guidelines for diagnosis, treatment and follow-up. Annals of 
oncology : official journal of the European Society for Medical Oncology / ESMO. Oct 2013;24 Suppl 6:vi24-32.

3. Wang W, Kryczek I, Dostal L, et al. Effector T Cells Abrogate Stroma-Mediated Chemoresistance in Ovarian Cancer. Cell. 519 2016;165(5):1092-1105. [PubMed: 27133165]

4. Lee N, Zakka LR, Mihm MC Jr., Schatton T. Tumour-infiltrating lymphocytes in melanoma prognosis and cancer immunotherapy. Pathology. 2 2016;48(2):177-187. [PubMed: 27020390]

5. Zhang L, Conejo-Garcia JR, Katsaros D, et al. Intratumoral T cells, recurrence, and survival in epithelial ovarian cancer. The New England journal of medicine. 116 2003;348(3):203-213. [PubMed: 12529460]

6. Curiel TJ, Coukos G, Zou L, et al. Specific recruitment of regulatory T cells in ovarian carcinoma fosters immune privilege and predicts reduced survival. Nature medicine. 9 2004;10(9):942-949.

7. Sato E, Olson SH, Ahn J, et al. Intraepithelial CD8+ tumor-infiltrating lymphocytes and a high $\mathrm{CD} 8+/$ regulatory $\mathrm{T}$ cell ratio are associated with favorable prognosis in ovarian cancer. Proceedings of the National Academy of Sciences of the United States of America. 1220 2005;102(51):1853818543. [PubMed: 16344461]

8. Jackson SR, Yuan J, Teague RM. Targeting CD8+ T-cell tolerance for cancer immunotherapy. Immunotherapy. 2014;6(7):833-852. [PubMed: 25290416]

9. Lin H, Wei S, Hurt EM, et al. Host expression of PD-L1 determines efficacy of PD-L1 pathway blockade-mediated tumor regression. The Journal of clinical investigation. 21 2018;128(2):805815. [PubMed: 29337305]

10. Zou W Immunosuppressive networks in the tumour environment and their therapeutic relevance. Nature reviews. Cancer. 4 2005;5(4):263-274. [PubMed: 15776005]

11. Postow MA, Callahan MK, Wolchok JD. Immune Checkpoint Blockade in Cancer Therapy. Journal of clinical oncology : official journal of the American Society of Clinical Oncology. 610 2015;33(17):1974-1982. [PubMed: 25605845]

12. Restifo NP, Dudley ME, Rosenberg SA. Adoptive immunotherapy for cancer: harnessing the T cell response. Nat Rev Immunol. 322 2012;12(4):269-281. [PubMed: 22437939]

13. Hutchinson L Immunotherapy: Harmonizing the immune response with a cancer vaccine. Nature reviews. Clinical oncology. 9 2012;9(9):487.

14. Topalian SL, Taube JM, Anders RA, Pardoll DM. Mechanism-driven biomarkers to guide immune checkpoint blockade in cancer therapy. Nature reviews. Cancer. May 2016;16(5):275-287.

15. Qureshi OS, Zheng Y, Nakamura K, et al. Trans-endocytosis of CD80 and CD86: a molecular basis for the cell-extrinsic function of CTLA-4. Science. 429 2011;332(6029):600-603. [PubMed: 21474713]

16. Shrikant P, Khoruts A, Mescher MF. CTLA-4 blockade reverses CD8+ T cell tolerance to tumor by a CD4+ T cell- and IL-2-dependent mechanism. Immunity. 10 1999;11(4):483-493. [PubMed: 10549630]

17. Dong H, Strome SE, Salomao DR, et al. Tumor-associated B7-H1 promotes T-cell apoptosis: a potential mechanism of immune evasion. Nature medicine. 8 2002;8(8):793-800.

18. Hui E, Cheung J, Zhu J, et al. T cell costimulatory receptor CD28 is a primary target for PD-1mediated inhibition. Science. 331 2017;355(6332):1428-1433. [PubMed: 28280247]

19. Zou W, Wolchok JD, Chen L. PD-L1 (B7-H1) and PD-1 pathway blockade for cancer therapy: Mechanisms, response biomarkers, and combinations. Sci Transl Med. 302 2016;8(328): 328 rv324.

20. Ribas A, Shin DS, Zaretsky J, et al. PD-1 Blockade Expands Intratumoral Memory T Cells. Cancer Immunol Res. 3 2016;4(3):194-203. [PubMed: 26787823]

21. Brahmer JR, Tykodi SS, Chow LQ, et al. Safety and activity of anti-PD-L1 antibody in patients with advanced cancer. The New England journal of medicine. 628 2012;366(26):2455-2465. [PubMed: 22658128]

22. Hamanishi J, Mandai M, Ikeda T, et al. Safety and Antitumor Activity of Anti-PD-1 Antibody, Nivolumab, in Patients With Platinum-Resistant Ovarian Cancer. Journal of clinical oncology : official journal of the American Society of Clinical Oncology. 121 2015;33(34):4015-4022. [PubMed: 26351349] 
23. Varga A, Piha-Paul SA, Ott PA, et al. Antitumor activity and safety of pembrolizumab in patients (pts) with PD-L1 positive advanced ovarian cancer: Interim results from a phase Ib study. Journal of Clinical Oncology. 2015;33(15_suppl):5510-5510.

24. Infante JR, Braiteh F, Emens LA, et al. Safety, clinical activity and biomarkers of atezolizumab (atezo) in advanced ovarian cancer (OC). Annals of Oncology. 2016;27(suppl_6):871P-871P.

25. Disis ML, Patel MR, Pant S, et al. Avelumab (MSB0010718C; anti-PD-L1) in patients with recurrent/refractory ovarian cancer from the JAVELIN Solid Tumor phase Ib trial: Safety and clinical activity. Journal of Clinical Oncology. 2016;34(15_suppl):5533-5533.

26. NCT01611558: Phase II Study of Ipilimumab Monotherapy in Recurrent Platinum-sensitive Ovarian Cancer - Study Results. https://clinicaltrials.gov/ct2/show/results/NCT01611558. Accessed 24 May 20162016.

27. Wolchok JD, Chiarion-Sileni V, Gonzalez R, et al. Overall Survival with Combined Nivolumab and Ipilimumab in Advanced Melanoma. The New England journal of medicine. 105 2017;377(14): 1345-1356. [PubMed: 28889792]

28. Goodman AM, Kato S, Bazhenova L, et al. Tumor Mutational Burden as an Independent Predictor of Response to Immunotherapy in Diverse Cancers. Molecular cancer therapeutics. 11 2017;16(11):2598-2608. [PubMed: 28835386]

29. Howitt BE, Shukla SA, Sholl LM, et al. Association of Polymerase e-Mutated and MicrosatelliteInstable Endometrial Cancers With Neoantigen Load, Number of Tumor-Infiltrating Lymphocytes, and Expression of PD-1 and PD-L1. JAMA Oncol. 12 2015;1(9):1319-1323. [PubMed: 26181000]

30. Yarchoan M, Hopkins A, Jaffee EM. Tumor Mutational Burden and Response Rate to PD-1 Inhibition. The New England journal of medicine. 1221 2017;377(25):2500-2501. [PubMed: 29262275]

31. Strickland KC, Howitt BE, Shukla SA, et al. Association and prognostic significance of BRCA1/2mutation status with neoantigen load, number of tumor-infiltrating lymphocytes and expression of PD-1/PD-L1 in high grade serous ovarian cancer. Oncotarget. 322 2016;7(12):13587-13598. [PubMed: 26871470]

32. Drew Y dJM Hong S-H, et al. An open-label, phase II basket study of olaparib and durvalumab (MEDIOLA): Results in germline BRCA-mutated (gBRCAm) platinum-sensitive relapsed (PSR) ovarian cancer (OC). Presented at: SGO Annual Meeting; March 24-27, 2018; New Orleans, LA Late-breaking abstract.

33. Ott PA, Hodi FS, Buchbinder EI. Inhibition of Immune Checkpoints and Vascular Endothelial Growth Factor as Combination Therapy for Metastatic Melanoma: An Overview of Rationale, Preclinical Evidence, and Initial Clinical Data. Frontiers in oncology. 2015;5:202. [PubMed: 26442214]

34. Voron T, Colussi O, Marcheteau E, et al. VEGF-A modulates expression of inhibitory checkpoints on CD8+ T cells in tumors. The Journal of experimental medicine. 29 2015;212(2):139-148. [PubMed: 25601652]

35. Bracci L, Schiavoni G, Sistigu A, Belardelli F. Immune-based mechanisms of cytotoxic chemotherapy: implications for the design of novel and rationale-based combined treatments against cancer. Cell death and differentiation. 1 2014;21(1):15-25. [PubMed: 23787994]

36. Galluzzi L, Zitvogel L, Kroemer G. Immunological Mechanisms Underneath the Efficacy of Cancer Therapy. Cancer Immunol Res. 11 2016;4(11):895-902. [PubMed: 27803050]

37. Mathios D, Kim JE, Mangraviti A, et al. Anti-PD-1 antitumor immunity is enhanced by local and abrogated by systemic chemotherapy in GBM. Sci Transl Med. 1221 2016;8(370):370ra180.

38. Langer CJ, Gadgeel SM, Borghaei H, et al. Carboplatin and pemetrexed with or without pembrolizumab for advanced, non-squamous non-small-cell lung cancer: a randomised, phase 2 cohort of the open-label KEYNOTE-021 study. The Lancet. Oncology 11 2016;17(11):1497-1508. [PubMed: 27745820]

39. Zitvogel L, Apetoh L, Ghiringhelli F, Kroemer G. Immunological aspects of cancer chemotherapy. Nat Rev Immunol. 1 2008;8(1):59-73. [PubMed: 18097448]

40. Emens LA, Middleton G. The interplay of immunotherapy and chemotherapy: harnessing potential synergies. Cancer Immunol Res. 5 2015;3(5):436-443. [PubMed: 25941355] 
41. Galluzzi L, Buque A, Kepp O, Zitvogel L, Kroemer G. Immunological Effects of Conventional Chemotherapy and Targeted Anticancer Agents. Cancer cell. 1214 2015;28(6):690-714. [PubMed: 26678337]

42. Galluzzi L, Vacchelli E, Bravo-San Pedro JM, et al. Classification of current anticancer immunotherapies. Oncotarget. 1230 2014;5(24):12472-12508. [PubMed: 25537519]

43. Kelderman S, Heemskerk B, Fanchi L, et al. Antigen-specific TIL therapy for melanoma: A flexible platform for personalized cancer immunotherapy. Eur J Immunol. 6 2016;46(6):13511360. [PubMed: 27005018]

44. Rosenberg SA, Restifo NP. Adoptive cell transfer as personalized immunotherapy for human cancer. Science. 43 2015;348(6230):62-68. [PubMed: 25838374]

45. Rosenberg SA, Yang JC, Sherry RM, et al. Durable complete responses in heavily pretreated patients with metastatic melanoma using T-cell transfer immunotherapy. Clinical cancer research : an official journal of the American Association for Cancer Research. 71 2011;17(13):4550-4557. [PubMed: 21498393]

46. Aoki Y, Takakuwa K, Kodama S, et al. Use of adoptive transfer of tumor-infiltrating lymphocytes alone or in combination with cisplatin-containing chemotherapy in patients with epithelial ovarian cancer. Cancer research. 41 1991;51(7):1934-1939. [PubMed: 2004379]

47. Freedman RS, Edwards CL, Kavanagh JJ, et al. Intraperitoneal adoptive immunotherapy of ovarian carcinoma with tumor-infiltrating lymphocytes and low-dose recombinant interleukin-2: a pilot trial. J Immunother Emphasis Tumor Immunol. 10 1994;16(3):198-210. [PubMed: 7834119]

48. Fujita K, Ikarashi H, Takakuwa K, et al. Prolonged disease-free period in patients with advanced epithelial ovarian cancer after adoptive transfer of tumor-infiltrating lymphocytes. Clinical cancer research : an official journal of the American Association for Cancer Research. 5 1995;1(5):501507. [PubMed: 9816009]

49. Abate-Daga D, Hanada K, Davis JL, Yang JC, Rosenberg SA, Morgan RA. Expression profiling of TCR-engineered $\mathrm{T}$ cells demonstrates overexpression of multiple inhibitory receptors in persisting lymphocytes. Blood. 822 2013;122(8):1399-1410. [PubMed: 23861247]

50. Rapoport AP, Stadtmauer EA, Binder-Scholl GK, et al. NY-ESO-1-specific TCR-engineered T cells mediate sustained antigen-specific antitumor effects in myeloma. Nature medicine. 8 2015;21(8):914-921.

51. Chapuis AG, Thompson JA, Margolin KA, et al. Transferred melanoma-specific CD8+ T cells persist, mediate tumor regression, and acquire central memory phenotype. Proceedings of the National Academy of Sciences of the United States of America. 320 2012;109(12):4592-4597. [PubMed: 22393002]

52. Fesnak AD, June CH, Levine BL. Engineered T cells: the promise and challenges of cancer immunotherapy. Nature reviews. Cancer. 823 2016;16(9):566-581. [PubMed: 27550819]

53. Matsuda T, Leisegang M, Park JH, et al. Induction of Neoantigen-specific Cytotoxic T Cells and Construction of T-cell Receptor-engineered T cells for Ovarian Cancer. Clinical cancer research : an official journal of the American Association for Cancer Research. 522018.

54. June CH, Sadelain M. Chimeric Antigen Receptor Therapy. The New England journal of medicine. 75 2018;379(1):64-73. [PubMed: 29972754]

55. June CH, O'Connor RS, Kawalekar OU, Ghassemi S, Milone MC. CAR T cell immunotherapy for human cancer. Science. 323 2018;359(6382):1361-1365. [PubMed: 29567707]

56. Kershaw MH, Westwood JA, Parker LL, et al. A phase I study on adoptive immunotherapy using gene-modified T cells for ovarian cancer. Clinical cancer research : an official journal of the American Association for Cancer Research. 1015 2006;12(20 Pt 1):6106-6115. [PubMed: 17062687]

57. Tanyi JL, Haas AR, Beatty GL, et al. Anti-mesothelin chimeric antigen receptor T cells in patients with epithelial ovarian cancer. Journal of Clinical Oncology. 2016;34(15_suppl):5511-5511.

58. Guo C, Manjili MH, Subjeck JR, Sarkar D, Fisher PB, Wang XY. Therapeutic cancer vaccines: past, present, and future. Adv Cancer Res. 2013;119:421-475. [PubMed: 23870514]

59. Thomas R, Al-Khadairi G, Roelands J, et al. NY-ESO-1 Based Immunotherapy of Cancer: Current Perspectives. Frontiers in immunology. 2018;9:947. [PubMed: 29770138] 
60. Diefenbach CS, Gnjatic S, Sabbatini P, et al. Safety and immunogenicity study of NY-ESO-1b peptide and montanide ISA-51 vaccination of patients with epithelial ovarian cancer in high-risk first remission. Clinical cancer research : an official journal of the American Association for Cancer Research. 51 2008;14(9):2740-2748. [PubMed: 18451240]

61. Odunsi K, Qian F, Matsuzaki J, et al. Vaccination with an NY-ESO-1 peptide of HLA class I/II specificities induces integrated humoral and $\mathrm{T}$ cell responses in ovarian cancer. Proceedings of the National Academy of Sciences of the United States of America. 731 2007;104(31):12837-12842. [PubMed: 17652518]

62. Sabbatini P, Tsuji T, Ferran L, et al. Phase I trial of overlapping long peptides from a tumor selfantigen and poly-ICLC shows rapid induction of integrated immune response in ovarian cancer patients. Clinical cancer research : an official journal of the American Association for Cancer Research. 121 2012;18(23):6497-6508. [PubMed: 23032745]

63. Odunsi K, Matsuzaki J, Karbach J, et al. Efficacy of vaccination with recombinant vaccinia and fowlpox vectors expressing NY-ESO-1 antigen in ovarian cancer and melanoma patients. Proceedings of the National Academy of Sciences of the United States of America. 410 2012;109(15):5797-5802. [PubMed: 22454499]

64. Odunsi K, Matsuzaki J, James SR, et al. Epigenetic potentiation of NY-ESO-1 vaccine therapy in human ovarian cancer. Cancer Immunol Res. Jan 2014;2(1):37-49.

65. Vermeij R, Leffers N, Hoogeboom BN, et al. Potentiation of a p53-SLP vaccine by cyclophosphamide in ovarian cancer: a single-arm phase II study. International journal of cancer. Journal international du cancer. 91 2012;131(5):E670-680. [PubMed: 22139992]

66. Martin Lluesma S, Wolfer A, Harari A, Kandalaft LE. Cancer Vaccines in Ovarian Cancer: How Can We Improve? Biomedicines. 53 2016;4(2).

67. Kalli KR, Block MS, Kasi PM, et al. Folate Receptor Alpha Peptide Vaccine generates immunity in Breast and Ovarian Cancer Patients. Clinical cancer research : an official journal of the American Association for Cancer Research. 3152018.

68. Jung NC, Lee JH, Chung KH, Kwak YS, Lim DS. Dendritic Cell-Based Immunotherapy for Solid Tumors. Transl Oncol. 6 2018;11(3):686-690. [PubMed: 29627706]

69. Loveland BE, Zhao A, White S, et al. Mannan-MUC1-pulsed dendritic cell immunotherapy: a phase I trial in patients with adenocarcinoma. Clinical cancer research : an official journal of the American Association for Cancer Research. 21 2006;12(3 Pt 1):869-877. [PubMed: 16467101]

70. Peethambaram PP, Melisko ME, Rinn KJ, et al. A phase I trial of immunotherapy with lapuleucel$\mathrm{T}$ (APC8024) in patients with refractory metastatic tumors that express HER-2/neu. Clinical cancer research : an official journal of the American Association for Cancer Research. 915 2009;15(18):5937-5944. [PubMed: 19723649]

71. Brossart P, Wirths S, Stuhler G, Reichardt VL, Kanz L, Brugger W. Induction of cytotoxic Tlymphocyte responses in vivo after vaccinations with peptide-pulsed dendritic cells. Blood. Nov 1 2000;96(9):3102-3108.

72. Chu CS, Boyer J, Schullery DS, et al. Phase I/II randomized trial of dendritic cell vaccination with or without cyclophosphamide for consolidation therapy of advanced ovarian cancer in first or second remission. Cancer immunology, immunotherapy : CII. 5 2012;61(5):629-641. [PubMed: 22021066]

73. Hernando JJ, Park TW, Kubler K, Offergeld R, Schlebusch H, Bauknecht T. Vaccination with autologous tumour antigen-pulsed dendritic cells in advanced gynaecological malignancies: clinical and immunological evaluation of a phase I trial. Cancer immunology, immunotherapy : CII. 3 2002;51(1):45-52. [PubMed: 11845259]

74. Tanyi JL, Bobisse S, Ophir E, et al. Personalized cancer vaccine effectively mobilizes antitumor T cell immunity in ovarian cancer. Sci Transl Med. 411 2018;10(436).

75. Senzer N, Barve M, Kuhn J, et al. Phase I trial of "bi-shRNAi(furin)/GMCSF DNA/autologous tumor cell" vaccine (FANG) in advanced cancer. Molecular therapy : the journal of the American Society of Gene Therapy. 3 2012;20(3):679-686. [PubMed: 22186789]

76. Li W, Tanikawa T, Kryczek I, et al. Aerobic Glycolysis Controls Myeloid-Derived Suppressor Cells and Tumor Immunity via a Specific CEBPB Isoform in Triple-Negative Breast Cancer. Cell Metab. 73 2018;28(1):87-103 e106. [PubMed: 29805099] 
77. Cui TX, Kryczek I, Zhao L, et al. Myeloid-derived suppressor cells enhance stemness of cancer cells by inducing microRNA101 and suppressing the corepressor CtBP2. Immunity. 919 2013;39(3):611-621. [PubMed: 24012420]

78. Peng D, Kryczek I, Nagarsheth N, et al. Epigenetic silencing of TH1-type chemokines shapes tumour immunity and immunotherapy. Nature. 1112 2015;527(7577):249-253. [PubMed: 26503055]

79. Guerriero JL, Sotayo A, Ponichtera HE, et al. Class IIa HDAC inhibition reduces breast tumours and metastases through anti-tumour macrophages. Nature. 316 2017;543(7645):428-432. [PubMed: 28273064]

80. Sheng W, LaFleur MW, Nguyen TH, et al. LSD1 Ablation Stimulates Anti-tumor Immunity and Enables Checkpoint Blockade. Cell. 726 2018;174(3):549-563 e519. [PubMed: 29937226]

81. Zhai L, Spranger S, Binder DC, et al. Molecular Pathways: Targeting IDO1 and Other Tryptophan Dioxygenases for Cancer Immunotherapy. Clinical cancer research : an official journal of the American Association for Cancer Research. 1215 2015;21(24):5427-5433. [PubMed: 26519060]

82. Zou W, Wolchok JD, Chen L. PD-L1 (B7-H1) and PD-1 Pathway Blockade for Cancer Therapy: Mechanisms, Response Biomarkers and Combinations. Sci Transl Med. 2016;8:328rv324.

83. Katsnelson A Mutations as munitions: Neoantigen vaccines get a closer look as cancer treatment. Nature medicine. 2 2016;22(2):122-124.

84. Ott PA, Hu Z, Keskin DB, et al. An immunogenic personal neoantigen vaccine for patients with melanoma. Nature. 713 2017;547(7662):217-221. [PubMed: 28678778]

85. Sahin U, Derhovanessian E, Miller M, et al. Personalized RNA mutanome vaccines mobilize polyspecific therapeutic immunity against cancer. Nature. 7 13 2017;547(7662):222-226. [PubMed: 28678784]

Surg Oncol Clin N Am. Author manuscript; available in PMC 2020 July 01. 


\section{KEY POINTS}

1. The application of immunotherapy in ovarian cancer is being tested.

2. Checkpoint blockade can restore the antitumor activity of effector $\mathrm{T}$ cells and mediate tumor regression.

3. Adoptive transfer of ex vivo-expanded tumor-specific $\mathrm{T}$ cells can directly increase the number of $\mathrm{CD} 8+$ effector $\mathrm{T}$ cells in tumor.

4. Cancer vaccine may activate tumor associated antigens-specific CD8+ T cell response through antigen presentation cells. 


\section{SYNOPSIS}

The phenotype and functionalities of the major immune cell subsets including myeloid cells, macrophages, dendritic cells, and T cells are altered in the ovarian cancer microenvironment. Immunosuppressive networks including inhibitory B7 family members and regulatory $\mathrm{T}$ cell-associated adenosine pathway have been defined in human ovarian cancer. In this review, the authors integrate emerging information on immunosuppressive mechanisms and $\mathrm{T}$ cell phenotype, and discuss strategies of immunotherapeutic and vaccine regimens. Finally, crucial points regarding design of immuno-oncology clinical trials are reviewed. 
Table 1.

Clinical studies of checkpoint blockade in EOC

\begin{tabular}{lllllll}
\hline Target & Agent & Phase & Trial identifier & Disease status & Patients & Response \\
\hline PD-1 & Nivolumab & II & UMIN000005714 & Platinum-resistant EOC & 20 & CR 2, PR 1, SD 6 \\
PD-1 & Pembrolizumab & I & & Recurrent EOC & 26 & CR 1, PR 2, SD 6 \\
PD-L1 & BMS-936559 & I & & Recurrent EOC & 17 & CR 0, PR 1, SD 3 \\
PD-L1 & Avelumab & I & Recurrent EOC & 124 & CR 0, PR 12, SD 55 \\
PD-L1 & Atezolizumab & I & & Recurrent EOC & 9 & CR 0, PR 2, SD 0 \\
CTLA-4 & Ipilimumab & I, II & & Recurrent EOC & 40 & $10 \%$ best response rate \\
\hline
\end{tabular}

NCT, National Clinical Trial; CR, complete response; PR, partial response; SD, stable disease 
Table 2.

Ongoing trials of checkpoint inhibitors in combination with other biological agents in EOC

\begin{tabular}{|c|c|c|c|c|c|}
\hline Combined agents & Combination & Treatment setting & Study population & Phase & $\begin{array}{c}\text { Trial } \\
\text { identifier }\end{array}$ \\
\hline \multirow{2}{*}{$\begin{array}{l}\text { Checkpoint } \\
\text { inhibitor }\end{array}$} & aCTLA-4 +aPD-1 & $\begin{array}{l}\text { Nivolumab vs nivolumab + } \\
\text { ipilimumab }\end{array}$ & Recurrent or pesistent EOC & IIa & \\
\hline & aPD-L1 +aCTLA-4 & Durvalumab + tremelimumab & & I & \\
\hline \multirow{4}{*}{ PARP inhibitor } & aCTLA-4 +PARPi & Tremelimumab + olaparib & $\begin{array}{l}\text { BRCA-deficient Ovarian } \\
\text { Cancer }\end{array}$ & $\mathrm{I} / \mathrm{II}$ & \\
\hline & aCTLA-4 +PARPi & $\begin{array}{l}\text { Tremelimumab vs tremelimumab + } \\
\text { olaparib }\end{array}$ & Recurrent or Persistent EOC & $\mathrm{I} / \mathrm{II}$ & \\
\hline & aPD-L1 +PARPi & $\begin{array}{l}\text { Durvalumab + olaparib vs } \\
\text { durvalumab + cediranib }\end{array}$ & $\begin{array}{l}\text { Recurrent EOC and germline } \\
\text { BRCA } 1 \text { or BRCA } 2 \text { mutation }\end{array}$ & $\mathrm{I} / \mathrm{IIa}$ & \\
\hline & $\begin{array}{l}\text { aCTLA4 +aPD-L1 } \\
\text { +PARPi }\end{array}$ & $\begin{array}{l}\text { Durvalumab + tremelimumab + } \\
\text { olaparib }\end{array}$ & $\begin{array}{l}\text { Recurrent EOC and germline } \\
\text { or somatic BRCA1 or } \\
\text { BRCA2 mutation }\end{array}$ & $\mathrm{I} / \mathrm{II}$ & \\
\hline \multirow[t]{5}{*}{ VEGF inhibitor } & aPD-L1+Bev & Atezolizumab + bevacizumab & $\begin{array}{l}\text { Recurrent Platinum Resistant } \\
\text { Ovarian Cancer }\end{array}$ & IIa & \\
\hline & aPD-L1 +PLD & $\begin{array}{l}\text { Avelumab+ Pegylated Liposomal } \\
\text { Doxorubicin }\end{array}$ & $\begin{array}{l}\text { Platinum Resistant/ } \\
\text { Refractory Ovarian Cancer }\end{array}$ & IIIa & \\
\hline & aPD-L1 + TC & Avelumab+ carboplatin + paclitaxel & $\begin{array}{l}\text { Advanced EOC with no } \\
\text { prior treatment }\end{array}$ & III & \\
\hline & $\mathrm{aPDL} 1+\mathrm{Bev}+\mathrm{TC} / \mathrm{PLD}$ & $\begin{array}{l}\text { atezolizumab + avastin + platinum- } \\
\text { based chemotherapy (Carboplatin } \\
\text { combined with gemcitabine or } \\
\text { paclitaxel or pegylated liposomal } \\
\text { doxorubicin) }\end{array}$ & $\begin{array}{l}\text { Recurrent platinum-sensitive } \\
\text { EOC }\end{array}$ & III & \\
\hline & $\mathrm{aPD}-\mathrm{L} 1+\mathrm{Bev}+\mathrm{TC}$ & $\begin{array}{l}\text { Atezolizumab+ Paclitaxel, } \\
\text { Carboplatin + Bevacizumab }\end{array}$ & $\begin{array}{l}\text { Newly-Diagnosed EOC with } \\
\text { no prior treatment }\end{array}$ & III & \\
\hline \multirow[t]{4}{*}{ Chemotherapy } & $\mathrm{aPD}-\mathrm{L} 1+\mathrm{Bev}+\mathrm{PLD}$ & $\begin{array}{l}\text { Atezolizumab + Pegylated } \\
\text { Liposomal Doxorubicin } \\
\text { Hydrochloride + or Bevacizumab }\end{array}$ & Platinum-resistant EOC & III & \\
\hline & aPD-L1 +PLD & Durvalumab + PLD & $\begin{array}{l}\text { Recurrent, Platinum- } \\
\text { Resistant Ovarian Cancer }\end{array}$ & $\mathrm{I} / \mathrm{II}$ & \\
\hline & $\mathrm{aPD}-1+\mathrm{TC}$ & $\begin{array}{l}\text { Pembrolizumab + paclitaxel + } \\
\text { carboplatin }\end{array}$ & Frontline Ovarian Cancer & II & \\
\hline & $\mathrm{aPD}-1+\mathrm{ddT}$ & $\begin{array}{l}\text { Pembrolizumab }+ \text { dose-dense } \\
\text { paclitaxel }\end{array}$ & $\begin{array}{l}\text { Recurrent, Platinum- } \\
\text { Resistant Ovarian Cancer }\end{array}$ & II & \\
\hline
\end{tabular}

aCTLA4, anti-CTLA-4; Bev, bevacizumab; ddT, dose-dense paclitaxel; NCT, National Clinical Trial; PARPi, PARP inhibitor; PLD, pegylated liposomal doxorubicin; TC, paclitaxel + carboplatin 
Table 3.

Completed clinical ACT therapy trials in EOC

\begin{tabular}{lcclll}
\hline Agent & Target antigens & $\begin{array}{l}\text { Trial } \\
\text { identifier }\end{array}$ & Disease status & Patients & Response \\
\hline TIL & - & - & Advanced or recurrent EOC & 17 & CR 8, PR 6, SD 1 \\
TIL & - & - & Recurrent EOC & 8 & CR 0, PR 0, SD 1 \\
TIL & - & - & $\begin{array}{l}\text { Advanced OC after surgery and } \\
\text { chemotherapy, 100\% disease-free }\end{array}$ & 13 & Improved overall survival \\
CAR & Folate receptor-a & & Recurrent FR EOC & 8 & No clinical benefit \\
CAR & mesothelin & EOC & 6 & $\begin{array}{l}\text { All patients showed stable disease at one } \\
\text { month after treatment }\end{array}$ \\
\hline
\end{tabular}

ACT, adoptive T-cell therapy; TIL, tumor-infiltrating lymphocytes; CAR, chimeric antigen receptor; FR, Folate receptor-a 
Table 4.

Ongoing ACT clinical trials in EOC

\begin{tabular}{|c|c|c|c|c|c|}
\hline Agent & Phase & Target antigen & Infused cells & Patients & $\begin{array}{l}\text { Trial } \\
\text { identifier }\end{array}$ \\
\hline TIL & II & - & short-term cultured autologous TIL & Metastasis ovarian cancer & \\
\hline TIL & I & - & Re-stimulated T cells & $\begin{array}{l}\text { Platinum-resistant high grade serous ovarian } \\
\text { cancer }\end{array}$ & \\
\hline TCR & $\mathrm{I}$ & MAGE-A4 & $\begin{array}{l}\text { MAGE-A4 specific TCR gene } \\
\text { transferred T lymphocytes }\end{array}$ & $\begin{array}{l}\text { HLA-A*24:02 positive MAGE-A4 expressing } \\
\text { EOC }\end{array}$ & \\
\hline TCR & $\mathrm{I}$ & NY-ESO-1 & $\begin{array}{l}\text { NY-ESO-1 specific TCR gene } \\
\text { transduced T lymphocytes }\end{array}$ & $\begin{array}{l}\text { HLA-A*02:01 or HLA-A*02:06 positive NY- } \\
\text { ESO- } 1 \text { expressing EOC }\end{array}$ & \\
\hline TCR & $\mathrm{I} / \mathrm{II}$ & NY-ESO-1 & $\begin{array}{l}\text { NY-ESO-1 (C259) transduced } \\
\text { autologous T cells }\end{array}$ & $\begin{array}{l}\text { Patients with HLA-A201, HLA-A205, and/or } \\
\text { HLA-A206 allele and whose tumor expresses } \\
\text { the NY-ESO-1 }\end{array}$ & \\
\hline TCR & I & NY-ESO-1 & Anti-NY ESO-1 TCR-transduced T cells & $\begin{array}{l}\text { Patients with HLA-A2+NY-ESO-1-expressing } \\
\text { tumor }\end{array}$ & \\
\hline CAR & $\mathrm{I} / \mathrm{II}$ & mesothelin & Anti-mesothelin CAR transduced PBL & $\begin{array}{l}\text { Metastatic or unresectable cancer that expresses } \\
\text { mesothelin }\end{array}$ & \\
\hline CAR & I & mesothelin & Anti-mesothelin CAR transduced T cells & $\begin{array}{l}\text { Refractory or relapsed mesothelin expressing } \\
\text { tumor }\end{array}$ & \\
\hline CAR & $\mathrm{I}$ & MUC16 & $\begin{array}{l}\text { 4H11-28z/fIL-12/EGFRt+ genetically- } \\
\text { modified T cells }\end{array}$ & EOC with MUC16ecto antigen expression & \\
\hline CAR & $\mathrm{I} / \mathrm{II}$ & EGFR & $\begin{array}{l}\text { anti-EGFR-CAR transduced autologous } \\
\mathrm{T} \text { cells }\end{array}$ & $\begin{array}{l}\text { Chemotherapy resistant or relapsed EOC with } \\
\text { EGFR expression }\end{array}$ & \\
\hline CAR & $\mathrm{I} / \mathrm{II}$ & ErbB2/Her2 & $\begin{array}{l}\text { anti-HER-2-CAR transduced autologous } \\
\mathrm{T} \text { cells }\end{array}$ & $\begin{array}{l}\text { Chemotherapy resistant or relapsed EOC with } \\
\text { Her } 2 \text { expression }\end{array}$ & \\
\hline CAR & $\mathrm{I}$ & CD133 & $\begin{array}{l}\text { anti-CD133-CAR vector-transduced T } \\
\text { cells }\end{array}$ & $\begin{array}{l}\text { Chemotherapy refractory or relapsed CD133- } \\
\text { positive EoC }\end{array}$ & \\
\hline
\end{tabular}

